\title{
PROPERTIES OF FUZZY DITANCE ON FUZZY SET
}

\author{
Dr. Jehad R. Kider, Aisha J. Hassan
}

Applied Mathematics, Department of Applied Science, University of Technology.

Applied Mathematics, Department of Applied Science, University of Technology

\begin{abstract}
In this paper we introduce the definition of fuzzy distance space on fuzzy set then we study and discuss several properties of this space after some illustrative examples are given. Furthermore we introduce the definition of fuzzy convergence, fuzzy Cauchy sequence of fuzzy point and fuzzy bounded fuzzy distance space .
\end{abstract}

\section{Keywords}

Fuzzy distance space on fuzzy; Fuzzy convergence; Fuzzy Cauchy sequence of fuzzy point and fuzzy bounded fuzzy distance space .

\section{Council for Innovative Research}

Peer Review Research Publishing System

Journal: JOURNAL OF ADVANCES IN MATHEMATICS

Vol .11, No.6

www.cirjam.com, editorjam@gmail.com 


\section{INTRODUCTION}

In 1965 fuzzy sets was introduced by Zadeh [20] many authors used this concept to formulate the notion of fuzzy metric on ordinary set $[1,2,3,7,8,9,10,13,15,16]$. In 1975 Kramosil and Michalek [14] introduce the definition of fuzzy metric on ordinary set which was called later the KM- fuzzy metric space. In 1994 George and Veermani [5] modified the KM-fuzzy metric space by taking instead of minimum function the binary operation t-norm which was called later GV-fuzzy metric space on ordinary set. In this paper we modified the definition of GV-fuzzy metric to a fuzzy space on fuzzy set $\widetilde{\mathrm{A}}$. This paper consist of three sections. In section two we introduce basic properties of fuzzy set and explain the difference between continuous and discrete fuzzy sets by example. After that we introduce the definition of fuzzy distance on fuzzy set then we give some examples to illustrate this notion. In section three we define fuzzy open fuzzy ball, fuzzy convergence of sequence of fuzzy points, fuzzy closed fuzzy set, fuzzy bounded fuzzy set, fuzzy dense fuzzy set and fuzzy Cauchy fuzzy sequence. It was proved that, let $\left(\tilde{A}, \widetilde{M},{ }^{*}\right)$ be a fuzzy distance space on the fuzzy set $\tilde{A}$ and let $\widetilde{B}$ be a subset of $\tilde{A}$. then $\widetilde{B}$ is a fuzzy dense in $\tilde{A}$ if and only if for every $a_{\alpha} \in \tilde{A}$ there is $b_{\beta} \in \widetilde{B}$ such that $\widetilde{M}\left(a_{\alpha}, b_{\beta}\right)>(1-\varepsilon)$ for some $0<\varepsilon<1$. (see Theorem 2.12).

\section{FUZZY DISTANCE SPACE ON A FUZZY SET}

\section{Definition 1.1:[20]}

Let $X$ be a nonempty set of elements, a fuzzy set $\widetilde{A}$ in $X$ is characterized by a membership function, $\mu_{\mathcal{A}}(X): X \rightarrow[0,1]$. Then we can write $\widetilde{\mathbb{A}}=\left\{\left(x, \mu_{\tilde{X}}(x)\right): x \in X, 0 \leq \mu_{\widetilde{A}}(x) \leq 1\right\}$.

We now recall an example of a continuous fuzzy set.

\section{Example 1.2:[17]}

Let $X=\mathbb{R}$ and let $\widetilde{\mathrm{A}}$ be a fuzzy set in $\mathbb{R}$ with membership function by:

$\mu_{X}(x)=\frac{1}{1+10 x^{2}}$.

\section{Definition 1.3:[4]}

Let $\tilde{A}$ and $\widetilde{B}$ be two fuzzy sets in $X$. then

1- $\tilde{\mathrm{A}} \subseteq \widetilde{\mathrm{B}}$ if and only if $\mu_{\tilde{\mathrm{A}}}(\mathrm{x}) \leq \mu_{\mathrm{B}}(\mathrm{x})$ for all $\mathrm{x} \in \mathrm{X}$

2- $\tilde{\mathrm{A}}=\widehat{\mathrm{B}}$ if and only if $\mu_{\tilde{\mathrm{A}}}(\mathrm{x})=\mu_{\mathrm{B}}(\mathrm{x})$ for all $\mathrm{x} \in \mathrm{X}$

3- $\tilde{\mathrm{C}}=\tilde{\mathrm{A}} \cup \widetilde{\mathrm{B}}$ if and only if $\mu_{\bar{C}}(\mathrm{x})=\mu_{\tilde{A}}(\mathrm{x}) \vee \mu_{\mathrm{B}}(\mathrm{x})$ for all $\mathrm{x} \in \mathrm{X}$

4- $\widetilde{\mathrm{D}}=\tilde{\mathrm{A}} \cap \widetilde{\mathrm{B}}$ if and only if $\mu_{\tilde{D}}(\mathrm{x})=\mu_{\tilde{\mathrm{A}}}(\mathrm{x}) \wedge \mu_{\mathrm{B}}(\mathrm{x})$ for all $\mathrm{x} \in \mathrm{X}$

5- $\mu_{\AA} c(x)=1-\mu_{\tilde{A}}(x)$ for all $x \in X$

\section{Definition 1.4:[17]}

If $\tilde{A}$ and $\widetilde{B}$ are fuzzy sets in a nonempty sets $X$ and $Y$ respectively then the

Cartesian product $\tilde{\mathrm{A}} \times \widetilde{\mathrm{B}}$ of $\tilde{\mathrm{A}}$ and $\widetilde{\mathrm{B}}$ is defined by:

$\mu_{\tilde{A} \times \mathbb{B}}(\mathrm{X}, \mathrm{y})=\mu_{\tilde{A}}(\mathrm{x}) \wedge \mu_{\mathrm{B}}(\mathrm{y})$ for all $(\mathrm{x}, \mathrm{y}) \in \mathrm{X} \times \mathrm{Y}$

\section{Definition 1.5:[19]}

A fuzzy point $p$ in $X$ is a fuzzy set with member

$$
\mathrm{p}(\mathrm{y})=\int_{1}^{\alpha} \quad \begin{array}{r}
\text { if } \mathrm{y}=\mathrm{x} \\
\text { Otherwise }
\end{array}
$$


For all $y$ in $X$ where $0<\alpha<1$. We denote this fuzzy point by $x_{\alpha}$. Two fuzzy points $x_{\alpha}$ and $y_{\beta}$ are said to be distinct if and only if $x \neq y$.

\section{Definition 1.6:[20]}

Let $\mathrm{x}_{\alpha}$ be a fuzzy point and $\tilde{\mathrm{A}}$ be a fuzzy set in $\mathrm{X}$. then $\mathrm{x}_{\alpha}$ is said to be in $\tilde{\mathrm{A}}$ or belongs to $\tilde{A}$ which is denoted by $x_{\alpha} \in \tilde{A}$ if and only if $\mu_{\tilde{A}}(x)>\alpha$.

\section{Definition 1.7:[11]}

Let $\mathrm{f}$ be a function from a nonempty set $\mathrm{X}$ into a nonempty set $\mathrm{Y}$. If $\widetilde{\mathrm{B}}$ is a fuzzy set in $Y$ then $\mathrm{f}^{-1}(\widetilde{B})$ is a fuzzy set in $X$ defined by:

$\mu_{f^{-1}(B)}(x)=\left(\mu_{\tilde{B} \circ} f\right)(x)$ for all $x$ in $X$. Also if $\tilde{A}$ is a fuzzy set in $X$ then $f(\tilde{A})$ is a fuzzy set in $Y$ defined by:

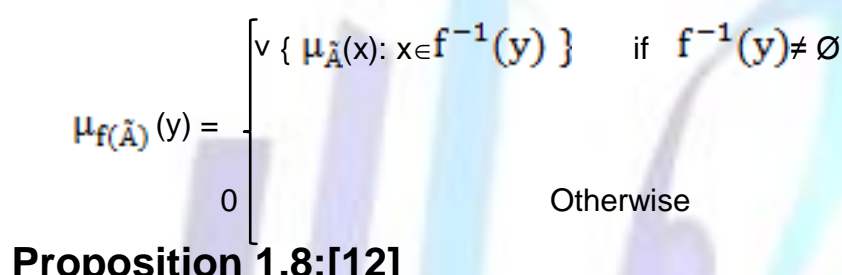

\section{Proposition 1.8:[12]}

Let $f: X \rightarrow Y$ be a function. Then for a fuzzy point $x_{\alpha \varepsilon}$ in $X, f\left(x_{\kappa \varepsilon}\right)$ is a fuzzy point in $Y$ and $f\left(x_{\kappa}\right)=(f(x))_{\alpha}$.

\section{Definition 1.9:[5]}

A binary operation $*:[0,1] \times[0,1] \rightarrow[0,1]$ is a continuous triangular norm (or simply t-norm ) if for all a, b, c, e $\in[0,1]$ the following conditions hold:

1

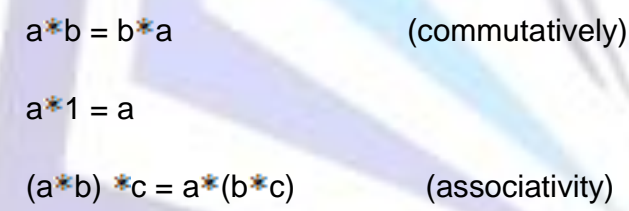
$a * 1=a$ (associativity)

2-

3- $\quad(a * b) * c=a *(b * c)$

4- If $a \leq c$ and $b \leq$ e then $a * b \leq c * e$.

\section{Example 1.10:[6]}

Define $a * b=a . b$, for all $a, b \in[0,1]$, where $a . b$ is the usual multiplication in $[0,1]$ then $*$ is a continuous t-norm.

\section{Example 1.11:[8]}

Define $a * b=\min \{a, b\}$ for all $a, b \in[0,1]$, it follows that $*$ is a continuous t-norm.

\section{Example 1.12:[10]}

Define $a * b=\max \{0, a+b-1\}$ for all $a, b \in[0,1]$, it follows that $*$ is a continuous t-norm.

\section{Remark 1.13:[5]}

For any $a>b$, we can find $c$ such that $a * c \geq b$ and for any $d$ we can find $q$ such that $q * q \geq d$, where $a, b, c, d$ and $q$ belong to $(0,1)$. 


\section{Now we introduce the definition of fuzzy distance space on fuzzy set Definition 1.14:}

Let $X$ be any set. The triple $(\tilde{A}, \widetilde{M}, *)$ is said to be a fuzzy distance space, where $\tilde{A}$ is an arbitrary fuzzy set in $\mathrm{X}$, * is continuous t-norm and $\widetilde{\mathrm{M}}$ is a fuzzy set on

$\tilde{\mathrm{A}} \times \tilde{\mathrm{A}} \rightarrow[0,1]$ satisfying the following conditions :

$\left(F M_{1}\right) \widetilde{M}\left(x_{\alpha}, y_{\beta}\right)>0$ for all $x_{\alpha}, y_{\beta} \in \tilde{A}$.

$\left(F M_{2}\right) \widetilde{M}\left(x_{\alpha}, y_{\beta}\right)=1$ if and only if $x_{\alpha}=. y_{\beta}$

$\left(F M_{3}\right) \widetilde{M}\left(x_{\alpha}, y_{\beta}\right)=\widetilde{M}\left(y_{\beta}, x_{\alpha}\right)$ for all $x_{\alpha}, y_{\beta} \in \tilde{A}$.

$\left(F M_{4}\right) \widetilde{M}\left(x_{\alpha}, y_{\beta}\right) * \widetilde{M}\left(y_{\beta}, z_{\sigma}\right) \leq \widetilde{M}\left(x_{\alpha}, z_{\sigma}\right)$ for all $x_{\alpha}, y_{\beta}, z_{\sigma} \in \tilde{A}$.

$\left(F M_{5}\right) \widetilde{M}\left(x_{\alpha}, y_{\beta}\right)$ : is a continuous fuzzy set for all $x_{\alpha}, y_{\beta}$.

\section{Remark 1.15:}

Condition $\left(\mathrm{FM}_{2}\right)$ means that $\widetilde{\mathrm{M}}\left(\mathrm{x}_{\kappa}, \mathrm{x}_{\kappa}\right)=1$ for all $\mathrm{x}_{\kappa} \in \tilde{\mathrm{A}}$ and $\widetilde{\mathrm{M}}\left(\mathrm{x}_{\kappa}, \mathrm{y}_{\beta}\right)<1$ for all $\mathrm{x}_{\alpha} \neq \mathrm{y}_{\beta}$ in $\tilde{\mathrm{A}}$.

\section{Remark 1.16:}

$\widetilde{\mathrm{M}}\left(\mathrm{x}_{\alpha}, \mathrm{y}_{\beta}\right)$ can be considered as the degree of nearness between $\mathrm{x}_{\alpha}$ and $\mathrm{y}_{\beta}$.

\section{Definition 1.17:}

Let $(\tilde{A}, \widetilde{M}, *)$ be a fuzzy distance space then $\widetilde{M}$ is continuous fuzzy set if whenever $\left(x_{n}, \alpha_{n}\right) \rightarrow x_{\alpha}$ and $\left(y_{n}, \beta_{n}\right) \rightarrow y_{\beta}$ in $\tilde{A}$ then $\widetilde{\mathrm{M}}\left(\left(\mathrm{x}_{\mathrm{n}}, \alpha_{\mathrm{n}}\right),\left(\mathrm{y}_{\mathrm{n}}, \beta_{\mathrm{n}}\right)\right) \rightarrow \widetilde{\mathrm{M}}\left(\mathrm{x}_{\alpha}, \mathrm{y}_{\beta}\right)$ that is $\lim _{\mathrm{n} \rightarrow \infty} \widetilde{\mathrm{M}}\left(\left(\mathrm{x}_{\mathrm{n}}, \alpha_{\mathrm{n}}\right),\left(\mathrm{y}_{\mathrm{n}}, \beta_{\mathrm{n}}\right)\right)=$ $\widetilde{\mathrm{M}}\left(\mathrm{x}_{\alpha}, \mathrm{y}_{\beta}\right)$.

\section{Lemma 1.18:}

Suppose that $(X, d)$ is an ordinary metric space and assume that $\tilde{A}$ is a fuzzy set in $X$. Define $d\left(X_{\alpha}, Y_{\beta}\right)=$ $\mathrm{d}(\mathrm{X}, \mathrm{Y})$ for all $\mathrm{X}_{\alpha}, \mathrm{Y}_{\beta} \in \tilde{\mathrm{A}}$. Then $(\tilde{\mathrm{A}}, \mathrm{d})$ is a metric space.

\section{Example 1.19:}

Let $X=\mathbb{R}$ and let $a * b=a . b$ for all $a, b \in[0,1]$.let $\tilde{A}$ be a fuzzy set in $\mathbb{R}$. Define

$\widetilde{\mathrm{M}}\left(\mathrm{x}_{\alpha}, \mathrm{y}_{\beta}\right)=\frac{1}{\exp \left|\mathrm{x}_{\alpha}-\mathrm{y}_{\beta}\right|}$ for all $\mathrm{x}_{\alpha}, \mathrm{y}_{\beta} \in \tilde{\mathrm{A}}$. Then $\left(\tilde{\mathrm{A}}, \widetilde{\mathrm{M}}_{z}\right)$ is a fuzzy distance space on the fuzzy set $\tilde{A}$.

\section{Proof:}

$\left(F M_{1}\right)$ It is clear that $\widetilde{M}\left(x_{\alpha}, y_{\beta}\right)>0$ for all $x_{\alpha}, y_{\beta} \in \tilde{A}$.

$\left(\mathrm{FM}_{2}\right)$ Assume that $\mathrm{x}_{\alpha}=\mathrm{y}_{\beta}$. Then this implies that $\left|\mathrm{x}_{\alpha}-\mathrm{y}_{\beta}\right|=0$

Hence $\frac{1}{\exp \left|x_{\alpha}-y_{\beta}\right|}=1$ implies that $\widetilde{\mathrm{M}}\left(\mathrm{x}_{\alpha}, \mathrm{y}_{\beta}\right)=1$ 
Conversely, assume that $\widetilde{\mathrm{M}}\left(\mathrm{x}_{\alpha}, \mathrm{y}_{\beta}\right)=1$. So $\frac{1}{\exp \left|\mathrm{x}_{\alpha}-\mathrm{y}_{\beta}\right|}=1$, which implies that $\exp \left|\mathrm{x}_{\alpha}-\mathrm{y}_{\beta}\right|=\mathrm{e}^{0}=1$. Hence $\left|\mathrm{x}_{\alpha}-\mathrm{y}_{\beta}\right|=0 \quad$ it follows $\mathrm{x}_{\alpha}=\mathrm{y}_{\beta}$.

Therefore $\widetilde{\mathrm{M}}\left(\mathrm{x}_{\alpha \varepsilon}, \mathrm{y}_{\beta}\right)=1$ if and only if $\mathrm{x}_{\alpha \varepsilon}=\mathrm{y}_{\beta}$

$\left(F M_{3}\right)$ Since $\left|x_{\alpha}-y_{\beta}\right|=\left|y_{\beta}-x_{\alpha \varepsilon}\right|$ for all $x_{\alpha}, y_{\beta} \in \tilde{A}$ it follows that

$\widetilde{\mathrm{M}}\left(\mathrm{x}_{\alpha}, \mathrm{y}_{\beta}\right)=\widetilde{\mathrm{M}}\left(\mathrm{y}_{\beta}, \mathrm{x}_{\kappa}\right)$ for all $\mathrm{x}_{\alpha}, \mathrm{y}_{\beta} \in \tilde{\mathrm{A}}$.

$\left(F M_{4}\right)$ To prove $\widetilde{\mathrm{M}}\left(\mathrm{x}_{\alpha}, \mathrm{y}_{\beta}\right) * \widetilde{\mathrm{M}}\left(\mathrm{y}_{\beta}, \mathrm{z}_{\sigma}\right) \leq \widetilde{\mathrm{M}}\left(\mathrm{x}_{\alpha}, \mathrm{z}_{\sigma}\right)$.

We know that for all $\mathrm{x}_{\alpha}, \mathrm{y}_{\beta}$, and $\mathrm{z}_{\sigma} \in \tilde{\mathrm{A}}$.

$$
\left|\mathrm{x}_{\alpha}-\mathrm{z}_{\sigma}\right| \leq\left|\mathrm{x}_{\alpha}-\mathrm{y}_{\beta}\right|+\left|\mathrm{y}_{\beta}-\mathrm{z}_{\sigma}\right|
$$

Thus $\exp \left|\mathrm{x}_{\alpha}-\mathrm{z}_{\sigma}\right| \leq \exp \left|\mathrm{x}_{\alpha}-\mathrm{y}_{\beta}\right| \cdot \exp \left|\mathrm{y}_{\beta}-\mathrm{z}_{\sigma}\right|$

Since $\exp \left(\mathrm{x}_{\alpha}\right)$ is an increasing function for all $\mathrm{x}_{\alpha}>0$

Therefore $\frac{1}{\exp \left|x_{\alpha}-z_{\sigma}\right|} \geq \frac{1}{\exp \left|x_{\alpha}-y_{\beta}\right|} * \frac{1}{\exp \left|y_{\beta}-z_{\sigma}\right|}$

Thus $\widetilde{\mathrm{M}}\left(\mathrm{x}_{\alpha}, \mathrm{z}_{\sigma}\right) \geq \widetilde{\mathrm{M}}\left(\mathrm{x}_{\kappa}, \mathrm{y}_{\beta}\right) * \widetilde{\mathrm{M}}\left(\mathrm{y}_{\beta}, \mathrm{z}_{\sigma}\right)$

$\left(\mathrm{FM}_{5}\right)$ Let $\left.\left\{\mathrm{x}_{\mathrm{n}}, \alpha_{\mathrm{n}}\right)\right\}$ and $\left\{\left(\mathrm{Y}_{\mathrm{n}}, \beta_{\mathrm{n}}\right)\right\}$ be two sequences in $\tilde{A}$ such that $\left(\mathrm{x}_{\mathrm{n}}, \alpha_{\mathrm{n}}\right) \rightarrow \mathrm{x}_{\kappa}$ and $\left(\mathrm{y}_{\mathrm{n}}, \beta_{\mathrm{n}}\right) \rightarrow \mathrm{y}_{\beta}$

Therefore $\lim _{n \rightarrow \infty} \widetilde{\mathrm{M}}\left(\left(\mathrm{x}_{\alpha}, \alpha_{\mathrm{n}}\right),\left(\mathrm{y}_{\mathrm{n}}, \beta_{\mathrm{n}}\right)\right)=\lim _{\mathrm{n} \rightarrow \infty} \frac{1}{\exp \left[\left(\mathrm{x}_{\mathrm{n},}, \alpha_{\mathrm{n}}\right)-\left(\mathrm{y}_{\mathrm{n}}, \beta_{\mathrm{n}}\right)\right]}$

$=\frac{1}{\lim _{n \rightarrow \infty} \exp \left|\left(x_{n}, \alpha_{n}\right)-\left(y_{n}, \beta_{n}\right)\right|}=\frac{1}{\exp \left(\lim _{n \rightarrow \infty}\left|\left(x_{n}, \alpha_{n}\right)-\left(y_{n}, \beta_{n}\right)\right|\right)}=\frac{1}{\exp \left|x_{\alpha}-y_{\beta}\right|}$

$=\widetilde{\mathrm{M}}\left(\mathrm{x}_{\alpha}, \mathrm{y}_{\beta}\right)$. That is $\left.\widetilde{\mathrm{M}}\left(\mathrm{x}_{\mathrm{n}}, \alpha_{\mathrm{n}}\right),\left(\mathrm{y}_{\mathrm{n}}, \beta_{\mathrm{n}}\right)\right) \rightarrow \widetilde{\mathrm{M}}\left(\mathrm{x}_{\alpha}, \mathrm{y}_{\beta}\right)$.

Hence $\widetilde{\mathrm{M}}$ is a continuous fuzzy set

\section{Remark 1.20:}

1-In example 1.19 we can replace $\mathbb{R}$ by any nonempty set $X$ and the usual metric on $\mathbb{R}$ by any metric $d$.

2-Example 1.19 is also a fuzzy metric space with the $t$-norm defined by $a * b=\min \{a, b\}$ for $a l l a, b \in[0,1]$.

\section{Example 1.21:}

Let $X=\mathbb{N}$ and $a * b=a . b$ for all $a, b \in[0,1]$ and let $\tilde{A}$ be $a$ fuzzy set in $X$

$$
\text { Define } \widetilde{\mathrm{M}}\left(\mathrm{x}_{\alpha}, \mathrm{y}_{\beta}\right)=-_{\frac{y}{x}}\left[\begin{array}{l}
\frac{x}{y} \\
\text { if } \mathrm{y} \leq \mathrm{x}
\end{array}\right.
$$

for all $\mathrm{x}_{\alpha \varepsilon}, \mathrm{y}_{\beta} \in \tilde{\mathrm{A}}$. Then $(\tilde{\mathrm{A}}, \widetilde{\mathrm{M}}, *)$ is a fuzzy distance space.

In the following example we show that not every fuzzy set on $\widetilde{A}^{2}$ is a fuzzy metric space on the fuzzy set $\tilde{A}$. 


\section{Example 1.22:}

Let $X=\mathbb{R}$ and let $\tilde{A}=[2, \infty]$ be a fuzzy set in $X$, consider the mapping

$\widetilde{\mathrm{M}}: \tilde{\mathrm{A}} \times \tilde{\mathrm{A}} \rightarrow[0,1]$ is defined by :

$\widetilde{\mathrm{M}}\left(\mathrm{a}_{\alpha}, \mathrm{b}_{\beta}\right)=\left\{\begin{array}{c}\text { if } \mathrm{a}=\mathrm{b} \\ \left(\frac{1}{\mathrm{a}}\right) \cdot \alpha+\left(\frac{1}{\mathrm{~b}}\right) \cdot \beta \quad \text { if } \mathrm{a} \neq \mathrm{b}\end{array}\right.$

Where $\alpha * \beta=\alpha$. $\beta$ for all $\alpha, \beta \in[0,1]$

\section{Proof:}

$\left(\mathrm{FM}_{4}\right)$ We show that $\widetilde{\mathrm{M}}\left(\mathrm{a}_{\alpha}, \mathrm{c}_{\sigma}\right) \geq \widetilde{\mathrm{M}}\left(\mathrm{a}_{\alpha}, \mathrm{b}_{\beta}\right) * \widetilde{\mathrm{M}}\left(\mathrm{b}_{\beta}, \mathrm{c}_{\sigma}\right)$ is not satisfied for all $\mathrm{a}_{\alpha}, \mathrm{b}_{\beta}, \mathrm{c}_{\sigma} \in \widetilde{\mathrm{A}}$. Let $\mathrm{a}_{\alpha}=10, \mathrm{~b}_{\beta}=3$ and $\mathrm{c}_{\sigma}=100$ where $\alpha=\frac{1}{a}, \beta=\frac{1}{\mathrm{~b}}, \sigma=\frac{1}{\mathrm{c}}$ Since $\mathrm{a} \neq \mathrm{b} \neq \mathrm{c}$

Then $\widetilde{\mathrm{M}}\left(\mathrm{a}_{\alpha}, \mathrm{b}_{\beta}\right)=\left(\frac{1}{\mathrm{a}}\right) \cdot \alpha+\left(\frac{1}{\mathrm{~b}}\right) \cdot \beta=\frac{1}{\mathrm{a}^{2}}+\frac{1}{\mathrm{~b}^{2}}=\frac{1}{100}+\frac{1}{9}=0.01+0.111=0.121$ And $\widetilde{\mathrm{M}}\left(\mathrm{b}_{\beta}, \mathrm{c}_{\sigma}\right)=\left(\frac{1}{\mathrm{~b}}\right) \cdot \beta+\left(\frac{1}{\mathrm{c}}\right) \cdot \sigma=\frac{1}{\mathrm{~b}^{2}}+\frac{1}{\mathrm{c}^{2}}=\frac{1}{9}+\frac{1}{10000}=0.111+0.0001=0.1112$

$\widetilde{\mathrm{M}}\left(\mathrm{a}_{\alpha}, \mathrm{c}_{\sigma}\right)=\left(\frac{1}{\mathrm{a}}\right) \cdot \alpha+\left(\frac{1}{\mathrm{c}}\right) \cdot \sigma=\frac{1}{\mathrm{a}^{2}}+\frac{1}{\mathrm{c}^{2}}=\frac{1}{100}+\frac{1}{10000}=0.01+0.0001=0.0101$

Therefore $\widetilde{\mathrm{M}}\left(\mathrm{a}_{\alpha}, \mathrm{b}_{\beta}\right) * \widetilde{\mathrm{M}}\left(\mathrm{b}_{\beta}, \mathrm{c}_{\sigma}\right)>\widetilde{\mathrm{M}}\left(\mathrm{a}_{\alpha}, \mathrm{c}_{\sigma}\right)$

Thus $\widetilde{\mathrm{M}}$ is not a fuzzy distance space

\section{Lemma 1.23:}

Let $(X, d)$ be an ordinary metric space and let $\tilde{A}$ be a fuzzy set in $X$. define $d\left(x_{\alpha}, y_{\beta}\right)=d(x, y)$ for all $x_{\alpha}, y_{\beta} \in \tilde{A}$. Then $(\tilde{A}, d)$ is a metric space.

\section{Proposition 1.24:}

Let $(X, d)$ be an ordinary metric space and let $a * b=a . b$ for all $a, b \in[0,1]$. Then by lemma $1.23,(\tilde{A}, d)$ is a metric space. Define $\widetilde{\mathrm{M}}_{\mathrm{d}}\left(\mathrm{x}_{\mathrm{\alpha}}, \mathrm{y}_{\beta}\right)=\frac{t}{t+d\left(\mathrm{x}_{\alpha}, y_{\beta}\right)}$, then $\left(\tilde{\mathrm{A}}, \widetilde{\mathrm{M}}_{\mathrm{d}}, *\right)$ is a fuzzy distance space and it is called the fuzzy metric on the fuzzy set $\tilde{A}$ induced by the metric $d$, where $t=\alpha \wedge \beta$.

\section{Proof:}

$\left(\mathrm{FM}_{1}\right)$ It is clear that $\widetilde{\mathrm{M}}_{\mathrm{d}}\left(\mathrm{x}_{\alpha}, \mathrm{y}_{\beta}\right)>0$ for all $\mathrm{x}_{\alpha}, \mathrm{y}_{\beta} \in \tilde{\mathrm{A}}$.

$\left(\mathrm{FM}_{2}\right)$ Assume that $\mathrm{x}_{\alpha}=\mathrm{y}_{\beta}$ then $\mathrm{d}\left(\mathrm{x}_{\alpha}, \mathrm{y}_{\beta}\right)=0$ so $\widetilde{\mathrm{M}}_{\mathrm{d}}\left(\mathrm{x}_{\alpha}, \mathrm{y}_{\beta}\right)=1$.

Conversely, assume that $\widetilde{\mathrm{M}}_{\mathrm{d}}\left(\mathrm{x}_{\alpha}, \mathrm{y}_{\beta}\right)=1$

So, $\frac{t}{t+d\left(x_{\alpha, y_{\beta}}\right)}=1$, implies $\mathrm{t}=\mathrm{t}+\mathrm{d}\left(\mathrm{x}_{\alpha}, \mathrm{y}_{\beta}\right)$

Or $\quad d\left(x_{\alpha}, y_{\beta}\right)=0$, so $x_{\alpha}=y_{\beta}$, thus $\widetilde{M}_{d}\left(x_{\alpha}, y_{\beta}\right)=1 \Leftrightarrow x_{\alpha}=y_{\beta}$.

$\left(\mathrm{FM}_{3}\right)$ Since $\mathrm{d}\left(\mathrm{x}_{\alpha}, \mathrm{y}_{\beta}\right)=\mathrm{d}\left(\mathrm{y}_{\beta}, \mathrm{x}_{\alpha}\right)$ so $\widetilde{\mathrm{M}}_{\mathrm{d}}\left(\mathrm{x}_{\alpha}, \mathrm{y}_{\beta}\right)=\widetilde{\mathrm{M}}_{\mathrm{d}}\left(\mathrm{y}_{\beta}, \mathrm{x}_{\kappa}\right)$. 
$\left(\mathrm{FM}_{4}\right)$ To prove $\widetilde{\mathrm{M}}_{\mathrm{d}}\left(\mathrm{x}_{\alpha}, \mathrm{y}_{\beta}\right) * \widetilde{\mathrm{M}}_{\mathrm{d}}\left(\mathrm{y}_{\beta},, \mathrm{z}_{\sigma}\right) \leq \widetilde{\mathrm{M}}_{\mathrm{d}}\left(\mathrm{x}_{\alpha}, \mathrm{z}_{\sigma}\right)$ notice that for all $\mathrm{x}_{\alpha}, \mathrm{y}_{\beta},, \mathrm{z}_{\sigma} \in \tilde{\mathrm{A}}$,

we have $\widetilde{\mathrm{M}}_{\mathrm{d}}\left(\mathrm{x}_{\alpha}, \mathrm{z}_{\sigma}\right)=\frac{\mathrm{t}}{\mathrm{t}+\mathrm{d}\left(\mathrm{x}_{\alpha,}, z_{\sigma}\right)} \geq \frac{\mathrm{t}}{\mathrm{t}+\mathrm{d}\left(\mathrm{x}_{\alpha}, y_{\beta}\right)+\mathrm{d}\left(\mathrm{y}_{\beta}, z_{\sigma}\right)}$

$$
\begin{aligned}
& \geq \frac{t}{t+d\left(x_{\alpha}, y_{\beta}\right)} \cdot \frac{t}{t+d\left(x_{\alpha} y_{\beta}\right)} \\
& =\widetilde{\mathrm{M}}_{d}\left(x_{\alpha}, y_{\beta}\right) * \widetilde{M}_{d}\left(y_{\beta}, z_{\sigma}\right)
\end{aligned}
$$

$\left(\mathrm{FM}_{5}\right)$ Let $\left\{\left(\mathrm{x}_{\mathrm{n}}, \alpha_{\mathrm{n}}\right)\right\}$ and $\left\{\left(\mathrm{y}_{\mathrm{n}}, \beta_{\mathrm{n}}\right)\right\}$ be two sequences of fuzzy points in $\tilde{A}$ such that $\left(\mathrm{x}_{\mathrm{n}}, \alpha_{\mathrm{n}}\right) \rightarrow \mathrm{x}_{\alpha},\left(\mathrm{y}_{\mathrm{n}}, \beta_{n}\right) \rightarrow \mathrm{y}_{\beta}$. Then $\lim _{\mathrm{n} \rightarrow \infty} \widetilde{\mathrm{M}}_{\mathrm{d}}\left(\left(\mathrm{x}_{\mathrm{n}}, \alpha_{\mathrm{n}}\right),\left(\mathrm{y}_{\mathrm{n}}, \beta_{\mathrm{n}}\right)\right)=\lim _{\mathrm{n} \rightarrow \infty} \frac{\mathrm{t}}{\mathrm{t}+\mathrm{d}\left(\left(\mathrm{x}_{\mathrm{n},}, \alpha_{\mathrm{n}}\right),\left(\mathrm{y}_{\mathrm{n}}, \beta_{\mathrm{n}}\right)\right)}=\frac{\mathrm{t}}{\mathrm{t}+\lim _{\mathrm{n} \rightarrow \infty} \mathrm{d}\left(\left(\mathrm{x}_{\mathrm{n}}, \alpha_{\mathrm{n}}\right)_{2}\left(\mathrm{y}_{\mathrm{n}}, \beta_{\mathrm{n}}\right)\right)}=\frac{\mathrm{t}}{\mathrm{t}+\mathrm{d}\left(\mathrm{x}_{\alpha}, y_{\beta}\right)}=$ $\widetilde{\mathrm{M}}_{\mathrm{d}}\left(\mathrm{x}_{\mathrm{ce}}, \mathrm{y}_{\beta}\right)$

That is $\widetilde{\mathrm{M}}_{\mathrm{d}}\left(\left(\mathrm{x}_{\mathrm{n}}, \alpha_{\mathrm{n}}\right),\left(\mathrm{y}_{\mathrm{n}}, \beta_{\mathrm{n}}\right)\right) \rightarrow \widetilde{\mathrm{M}}_{\mathrm{d}}\left(\mathrm{x}_{\alpha}, \mathrm{y}_{\beta}\right)$. Hence $\widetilde{\mathrm{M}}$ is a continuous fuzzy set

\section{Remark 1.25:}

Let $(\tilde{\mathrm{A}}, \widetilde{\mathrm{M}}, *)$ be a fuzzy distance space. Then $\left.\widetilde{\mathrm{M}}\left(\mathrm{x}_{1}, \alpha_{1}\right),\left(\mathrm{x}_{\mathrm{n}}, \alpha_{\mathrm{n}}\right)\right) \geq \widetilde{\mathrm{M}}\left(\left(\mathrm{x}_{1}, \alpha_{1}\right),\left(\mathrm{x}_{2}, \alpha_{2}\right)\right)$

$\left.* \widetilde{\mathrm{M}}\left(\left(\mathrm{x}_{2}, \alpha_{2}\right),\left(\mathrm{x}_{3}, \alpha_{3}\right)\right) * \ldots * \widetilde{\mathrm{M}}_{(}\left(\mathrm{x}_{\mathrm{n}-1}, \alpha_{\mathrm{n}-1}\right),\left(\mathrm{x}_{\mathrm{n}}, \alpha_{\mathrm{n}}\right)\right)$.

\section{Fuzzy Convergence, Fuzzy Cauchy Sequences, Fuzzy}

\section{bounded, Fuzzy open and Fuzzy closed Fuzzy sets}

In this section Ã will be a fuzzy set in the nonempty set $X$.

\section{Definition 2.1:}

Let $(\tilde{A}, \widetilde{\mathrm{M}}, *)$ be a fuzzy distance space on the fuzzy set $\tilde{\mathrm{A}}$, we define $\widetilde{\mathrm{B}}\left(\mathrm{x}_{\mathrm{e}}, \mathrm{r}\right)=\left\{\mathrm{y}_{\beta} \in \tilde{\mathrm{A}}: \widetilde{\mathrm{M}}\left(\mathrm{x}_{\alpha}, \mathrm{y}_{\beta}\right)>\right.$

$(1-r)\}$ then $\widetilde{B}\left(x_{\alpha \varepsilon}, r\right)$ is called an fuzzy open fuzzy ball with center the fuzzy point $x_{\alpha \varepsilon} \in \tilde{A}$ and radius

$$
0<\mathrm{r}<1 \text {. }
$$

\section{Proposition 2.2:}

Let $\widetilde{\mathrm{B}}\left(\mathrm{x}_{\alpha}, \mathrm{r}_{1}\right)$ and $\widetilde{\mathrm{B}}\left(\mathrm{x}_{\alpha}, \mathrm{r}_{2}\right)$ be two fuzzy open fuzzy balls with the same center $\mathrm{x}_{\alpha} \in \tilde{\mathrm{A}}$ and with radiuses $\mathrm{r}_{1}, \mathrm{r}_{2} \in(0,1)$. Then we either have $\widetilde{\mathrm{B}}\left(\mathrm{x}_{\mathrm{a}}, \mathrm{r}_{1}\right) \subseteq \widetilde{\mathrm{B}}\left(\mathrm{x}_{\mathrm{a}}, \mathrm{r}_{2}\right)$ or

$\widetilde{\mathrm{B}}\left(\left(\mathrm{x}_{\mathrm{a}}, \mathrm{r}_{2}\right) \subseteq \widetilde{\mathrm{B}}\left(\mathrm{x}_{\mathrm{a}}, \mathrm{r}_{1}\right)\right.$.

\section{Proof:}

Let $\mathrm{x}_{\alpha \varepsilon} \in \tilde{\mathrm{A}}$ and consider the fuzzy open fuzzy balls $\widetilde{\mathrm{B}}\left(\mathrm{x}_{\alpha}, \mathrm{r}_{1}\right)$ and $\widetilde{\mathrm{B}}\left(\mathrm{x}_{\alpha}, \mathrm{r}_{2}\right)$ with $\mathrm{r}_{1}, \mathrm{r}_{2} \in(0,1)$. If $\mathrm{r}_{1}=\mathrm{r}_{2}$ then the proposition holds.

Next, we assume that $r_{1} \neq r_{2}$, we may assume without loss of generality that

$\mathrm{r}_{1}<\mathrm{r}_{2}$ this implies that $\left(1-\mathrm{r}_{2}\right)<\left(1-\mathrm{r}_{1}\right)$.

Now let $\mathrm{y}_{\beta} \in \widetilde{\mathrm{B}}\left(\mathrm{x}_{\alpha}, \mathrm{r}_{1}\right)$, it follows that $\widetilde{\mathrm{M}}\left(\mathrm{y}_{\beta}, \mathrm{x}_{\alpha}\right)>\left(1-\mathrm{r}_{1}\right)$. So $\widetilde{\mathrm{M}}\left(\mathrm{y}_{\beta}, \mathrm{x}_{\alpha}\right)>$

(1- $\left.\mathrm{r}_{2}\right)$. Hence $\mathrm{y}_{\beta} \in \widetilde{\mathrm{B}}\left(\mathrm{x}_{\alpha}, \mathrm{r}_{2}\right)$. This shows that $\widetilde{\mathrm{B}}\left(\mathrm{x}_{\alpha}, \mathrm{r}_{1}\right) \subseteq \widetilde{\mathrm{B}}\left(\mathrm{x}_{\alpha}, \mathrm{r}_{2}\right)$. By

assuming that $r_{2}<r_{1}$.

We can similarly show that $\widetilde{\mathrm{B}}\left(\mathrm{x}_{\alpha}, \mathrm{r}_{2}\right) \subseteq \widetilde{\mathrm{B}}\left(\left(\mathrm{x}_{\alpha}, \mathrm{r}_{1}\right)\right.$ 


\section{Definition 2.3:}

A sequence $\left\{\left(\mathrm{x}_{\mathrm{n}}, \alpha_{\mathrm{n}}\right)\right\}$ of fuzzy points in a fuzzy distance space $(\tilde{\mathrm{A}}, \widetilde{\mathrm{M}}, *)$ is said to be fuzzy converges to a fuzzy pointx $\mathrm{x}_{\alpha} \in \tilde{\mathrm{A}}$ if for all $0<\varepsilon<1$, there exists a positive number $N$ such that, $\widetilde{M}\left(\left(x_{n}, \alpha_{n}\right), x_{a}\right)>(1-\varepsilon)$ for all $n \geq N$.

\section{Definition 2.4:}

A sequence $\left\{\left(\mathrm{x}_{\mathrm{n}}, \alpha_{\mathrm{n}}\right)\right\}$ of fuzzy points in a fuzzy distance space $(\tilde{\mathrm{A}}, \widetilde{\mathrm{M}}, *)$ is said to be fuzzy converges to a fuzzy point $\mathrm{x}_{\mathrm{\alpha}} \in \tilde{\mathrm{A}}$ if $\lim _{n \rightarrow \infty} \widetilde{M}\left(\left(x_{n}, \alpha_{n}\right), x_{c e}\right)=1$.

\section{Theorem 2.5:}

Definition 2.3 and definition 2.4 are equivalent.

\section{Proof:}

Suppose that the sequence $\left\{\left(\mathrm{x}_{\mathrm{n}}, \alpha_{\mathrm{n}}\right)\right\}$ fuzzy converges to $\mathrm{x}_{\mathrm{e}}$ in sense of definition 2.3 then for all $0<\mathrm{r}<1$ there exists a positive number $\mathrm{N}$ such that $\widetilde{\mathrm{M}}\left(\left(\mathrm{x}_{\mathrm{n}}, \alpha_{\mathrm{n}}\right), \mathrm{x}_{\kappa}\right)>(1-\mathrm{r})$ for all $\mathrm{n} \geq \mathrm{N}$ and hence $\left[1-\widetilde{\mathrm{M}}\left(\left(\mathrm{x}_{\mathrm{n}}, \alpha_{\mathrm{n}}\right), \mathrm{x}_{\kappa}\right)\right]<\mathrm{r}$. Therefore $\widetilde{\mathrm{M}}\left(\left(\mathrm{x}_{\mathrm{n}}, \alpha_{\mathrm{n}}\right)\right.$, $\left.\mathrm{x}_{\alpha}\right)$ converges to 1 as $\mathrm{n}$ tends to $\infty$. Conversely, assume that $\widetilde{\mathrm{M}}\left(\left(\mathrm{x}_{\mathrm{n}}, \alpha_{\mathrm{n}}\right), \mathrm{x}_{\alpha}\right)$ converges to 1 as $\mathrm{n}$ tends to $\infty$. Then for $0<r<1$ there exists a positive integer $\mathrm{N}$ such that,

$\left[1-\widetilde{M}\left(\left(x_{n}, \alpha_{n}\right), x_{\alpha}\right)\right]<r$ for all $n \geq N$. It follows that $\widetilde{M}\left(\left(x_{n}, \alpha_{n}\right), x_{\alpha}\right)>(1-r)$ for all $n \geq N$. Hence $\left\{\left(x_{n}, \alpha_{n}\right)\right\}$ fuzzy converges to $\mathrm{x}_{\mathrm{a}}$ in sense of Definition of 2.4

\section{Proposition 2.6:}

Let $(X, d)$ be a metric space and let $\left(\tilde{A}, \widetilde{M}_{d}, *\right)$ be the fuzzy distance space induced by $d$. Let $\left\{\left(\mathrm{x}_{\mathrm{n}}, \alpha_{\mathrm{n}}\right)\right\}$ be a sequence of fuzzy points in $\tilde{\mathrm{A}}$. Then $\left\{\left(\mathrm{x}_{\mathrm{n}}, \alpha_{\mathrm{n}}\right)\right\}$ converges to $\mathrm{x}_{\alpha} \in \tilde{\mathrm{A}}$ in $(\tilde{\mathrm{A}}, \mathrm{d})$ if and only if $\left\{\left(\mathrm{x}_{\mathrm{n}}, \alpha_{\mathrm{n}}\right)\right\}$ fuzzy converges to $\mathrm{x}_{\alpha}$ in $\left(\tilde{\mathrm{A}}, \widetilde{\mathrm{M}}_{\mathrm{d}}, *\right)$.

\section{Proof:}

Suppose that $\left\{\left(\mathrm{x}_{\mathrm{n}}, \alpha_{\mathrm{n}}\right)\right\}$ converges to $\mathrm{x}_{\alpha} \in \tilde{\mathrm{A}}$ in $(\tilde{\mathrm{A}}, \mathrm{d})$ it follows that $\lim _{n \rightarrow \infty} d\left(\left(x_{n}, \alpha_{n}\right), x_{\alpha}\right)=0$

Now,

$$
\lim _{n \rightarrow \infty} \widetilde{M}_{d}\left(\left(x_{n}, \alpha_{n}\right), x_{\alpha}\right)=\lim _{n \rightarrow \infty} \frac{t}{t+d\left(\left(x_{n}, a_{n}\right) x_{\alpha}\right)}=\frac{t}{t+\lim _{n \rightarrow \infty} d\left(\left(x_{n}, a_{n}\right) x_{\alpha}\right)}=1
$$

Hence $\left\{\left(\mathrm{x}_{\mathrm{n}}, \alpha_{\mathrm{n}}\right)\right\}$ fuzzy converges to $\mathrm{x}_{\alpha}$ in $\left(\tilde{\mathrm{A}}, \widetilde{\mathrm{M}}_{\mathrm{d}}, *\right)$, where $\mathrm{t}=\min \left\{\alpha, \alpha_{\mathrm{n}}\right\}$

Conversely, assume that $\left\{\left(\mathrm{x}_{\mathrm{n}}, \alpha_{\mathrm{n}}\right)\right\}$ fuzzy converge to $\mathrm{x}_{\alpha}$ in $\left(\tilde{\mathrm{A}}, \widetilde{\mathrm{M}}_{\mathrm{d}}, *\right)$, it

follows that $\lim _{\mathrm{n} \rightarrow \infty} \widetilde{\mathrm{M}}_{\mathrm{d}}\left(\left(\mathrm{x}_{\mathrm{n}}, \alpha_{\mathrm{n}}\right), \mathrm{x}_{\alpha}\right)=1$

Now, $\quad \lim _{n \rightarrow \infty} \frac{t}{t+d\left(\left(x_{n}, \alpha_{n}\right) x_{\alpha}\right)}=1$, where $t=\min \left\{\alpha, \alpha_{n}\right\}$ which implies

that $\frac{t}{t+\lim _{n \rightarrow \infty} d\left(\left(x_{n y} \alpha_{n}\right) x_{\alpha}\right)}=1$, so $t+\lim _{n \rightarrow \infty} d\left(\left(x_{n}, \alpha_{n}\right), x_{\alpha}\right)=t$, it follows

that $\lim _{\mathrm{n} \rightarrow \infty} \mathrm{d}\left(\left(\mathrm{x}_{\mathrm{n}}, \alpha_{\mathrm{n}}\right), \mathrm{x}_{\alpha}\right)=\mathrm{t}-\mathrm{t}=0$

Hence $\left\{\left(\mathrm{x}_{\mathrm{n}}, \alpha_{\mathrm{n}}\right)\right\}$ fuzzy converges to $\mathrm{x}_{\alpha}$ in $(\tilde{\mathrm{A}}, \mathrm{d})$

Definition 2.7: A fuzzy subset $\widetilde{\mathrm{C}}$ of a fuzzy distance space $(\tilde{\mathrm{A}}, \widetilde{\mathrm{M}}, *)$ is said to be fuzzy open if it contains a fuzzy ball about each of its fuzzy points. A fuzzy subset $\widetilde{\mathrm{D}}$ of 
$(\tilde{\mathrm{A}}, \widetilde{\mathrm{M}}, *)$ is said to be fuzzy closed if its complement is fuzzy open that is $\widetilde{\mathrm{D}}^{\mathrm{c}}=\tilde{\mathrm{A}}-\widetilde{\mathrm{D}}$ is fuzzy open.

\section{Theorem 2.8:}

Every fuzzy open fuzzy ball in a fuzzy distance space $(\tilde{\mathrm{A}}, \widetilde{\mathrm{M}}, *)$ on a fuzzy set $\tilde{\mathrm{A}}$ is a fuzzy open fuzzy set.

\section{Proof:}

Consider a fuzzy open fuzzy ball $\widetilde{\mathrm{B}}\left(\mathrm{x}_{\alpha}, r\right)$ where $\mathrm{x}_{\alpha \varepsilon} \in \tilde{\mathrm{A}}$ and $0<\mathrm{r}<1$. let $\mathrm{y}_{\beta} \in \widetilde{\mathrm{B}}\left(\mathrm{x}_{\alpha}, r\right) \quad$ implies $\widetilde{\mathrm{M}}\left(\mathrm{x}_{\alpha}, \mathrm{y}_{\beta}\right)>(1-r)$, put $t$ $=\widetilde{\mathrm{M}}\left(\mathrm{x}_{\alpha}, \mathrm{y}_{\beta}\right)>(1-r)$, then we can find $\mathrm{s}, 0<\mathrm{s}<1$, such that $\mathrm{t}>(1-\mathrm{s})>(1-r)$. Now for a given $\mathrm{t}$ and $\mathrm{s}$ such that $\mathrm{t}>$ (1- $\mathrm{s}$ ), we can find $0<\mathrm{r}_{1}<1$ such that ( $\mathrm{t}(1-\mathrm{s})$ by Remark 1.13 , now consider the fuzzy ball $\widetilde{\mathrm{B}}\left(\mathrm{y}_{\beta}, 1-\mathrm{r}_{1}\right)$, we claim $\widetilde{\mathrm{B}}\left(\mathrm{y}_{\beta}, 1-\mathrm{r}_{1}\right) \subseteq \widetilde{\mathrm{B}}\left(\mathrm{x}_{\alpha}, r\right)$. Let $\mathrm{z}_{\sigma} \in \widetilde{\mathrm{B}}\left(\mathrm{y}_{\beta}, 1-\mathrm{r}_{1}\right)$ so $\widetilde{\mathrm{M}}\left(\mathrm{y}_{\beta}, \mathrm{z}_{\sigma}\right)>\mathrm{r}_{1}$. Therefore $\widetilde{\mathrm{M}}\left(\mathrm{x}_{\alpha}, \mathrm{z}_{\sigma}\right) \geq \widetilde{\mathrm{M}}\left(\mathrm{x}_{\alpha}, \mathrm{y}_{\beta}\right) * \widetilde{\mathrm{M}}\left(\mathrm{y}_{\beta}, \mathrm{z}_{\sigma}\right)$

$\widetilde{\mathrm{M}}\left(\mathrm{x}_{\alpha}, \mathrm{z}_{\sigma}\right) \geq\left(\mathrm{t} * \mathrm{r}_{1}\right) \geq(1-\mathrm{s})>(1-\mathrm{r})$. Hence $\mathrm{z}_{\sigma} \in \widetilde{\mathrm{B}}\left(\mathrm{x}_{\alpha}, r\right)$ so $\widetilde{\mathrm{B}}\left(\mathrm{y}_{\beta}, 1-\mathrm{r}_{1}\right) \subseteq \widetilde{\mathrm{B}}\left(\mathrm{x}_{\alpha}, r\right)$

\section{Definition 2.9:}

Let ( $\tilde{\mathrm{A}}, \widetilde{\mathrm{M}}$,*) be a fuzzy distance space on a fuzzy set $\tilde{\mathrm{A}}$ and let $\tilde{\mathrm{C}} \subset \tilde{\mathrm{A}}$ then the fuzzy closure of $\tilde{\mathrm{C}}$ is denoted by $\overline{\widetilde{C}}$ or $\operatorname{FCL}(\tilde{\mathrm{C}})$ and is defined to be the smallest fuzzy closed fuzzy set contains $\tilde{\mathrm{C}}$.

\section{Definition 2.10:}

A fuzzy subset $\widetilde{\mathrm{C}}$ of a fuzzy distance space $(\tilde{\mathrm{A}}, \widetilde{\mathrm{M}}, *)$ on a fuzzy set $\tilde{A}$ is said to be fuzzy dense in $\tilde{\mathrm{A}}$ if $\overline{\mathrm{C}}=\tilde{\mathrm{A}}$.

\section{Lemma 2.11:}

Let $\tilde{\mathrm{C}}$ be a fuzzy subset of $\tilde{\mathrm{A}}$ and let $(\tilde{\mathrm{A}}, \widetilde{\mathrm{M}}, *)$ be a fuzzy distance space on the fuzzy set $\tilde{\mathrm{A}}$ then $\mathrm{a}_{\mathrm{c}} \in \overline{\widetilde{C}}$ if and only if there is a sequence $\left\{\left(\mathrm{a}_{\mathrm{n}}, \alpha_{\mathrm{n}}\right)\right\}$ in $\tilde{\mathrm{C}}$ such that $\left(\mathrm{a}_{\mathrm{n}}, \alpha_{\mathrm{n}}\right) \rightarrow \mathrm{a}_{\mathrm{a}}$, where $\alpha, \alpha_{\mathrm{n}} \in[0,1]$.

\section{Proof:}

Let $\mathrm{a}_{\alpha} \in \overline{\mathrm{C}}$, if $\mathrm{a}_{\alpha} \in \widetilde{\mathrm{C}}$ then we take sequence of that type is $\left(\mathrm{a}_{\alpha}, \mathrm{a}_{\alpha}, \mathrm{a}_{\alpha}, \ldots, \mathrm{a}_{\alpha}, \ldots\right)$. If $\mathrm{a}_{\alpha} \notin \widetilde{\mathrm{C}}$, it is a limit fuzzy point of $\tilde{\mathrm{C}}$. Hence we construct the sequence $\left(\mathrm{a}_{\mathrm{n}}, \alpha_{\mathrm{n}}\right) \in \widetilde{\mathrm{C}}$ by $\widetilde{\mathrm{M}}\left(\left(\mathrm{a}_{\mathrm{n}}, \alpha_{\mathrm{n}}\right), \mathrm{a}_{\alpha}\right)>\left(1-\frac{1}{\mathrm{n}}\right)$ for each $\mathrm{n}=1,2,3, \ldots$. The fuzzy ball $\widetilde{\mathrm{B}}\left(\mathrm{a}_{\alpha}, \frac{1}{\mathrm{n}}\right)$ contains $\left(a_{n}, \alpha_{n}\right) \in \widetilde{C}$ and $\left(a_{n}, \alpha_{n}\right) \rightarrow a_{\alpha}$ because $\lim _{n \rightarrow \infty} \widetilde{M}\left(\left(a_{n}, \alpha_{n}\right), a_{a c}\right)=1$. Conversely if $\left\{\left(a_{n}, \alpha_{n}\right)\right\}$ in $\widetilde{\mathrm{C}}$ and $\left(a_{n}, \alpha_{n}\right) \rightarrow a_{\alpha}$ then $a_{\alpha} \in \tilde{C}$, or every neighborhood of $a_{\alpha}$ contains fuzzy points $\left(a_{n}, \alpha_{n}\right) \neq a_{\alpha}$, so that $a_{\alpha}$ is a fuzzy limit of $\tilde{\mathrm{C}}$, hence $\mathrm{a}_{\alpha} \in \overline{\mathrm{C}}$ by the definition of the fuzzy closure

\section{Theorem 2.12:}

Let $\tilde{\mathrm{C}}$ be a fuzzy subset of a fuzzy distance space $(\tilde{\mathrm{A}}, \widetilde{\mathrm{M}}, *)$ then $\widetilde{\mathrm{C}}$ is fuzzy dense in $\tilde{\mathrm{A}}$ if and only if for every $\mathrm{x}_{\alpha} \in \tilde{\mathrm{A}}$ there is $\mathrm{a}_{\beta} \in \tilde{\mathrm{C}}$ such that $\widetilde{\mathrm{M}}\left(\mathrm{x}_{\alpha}, \mathrm{a}_{\beta}\right)>(1-\varepsilon)$ for some $0<\varepsilon<1$.

\section{Proof:}

Suppose that $\tilde{\mathrm{C}}$ is fuzzy dense in $\tilde{\mathrm{A}}$ and $\mathrm{x}_{\alpha} \in \tilde{\mathrm{A}}$ so $\mathrm{x}_{\alpha \varepsilon} \in \overline{\widetilde{C}}$ and by Lemma 2.11 there is a sequence $\left\{\left(\mathrm{a}_{\mathrm{n}}, \beta_{\mathrm{n}}\right)\right\} \in \tilde{\mathrm{C}}$ such that $\left(a_{n}, \beta_{n}\right) \rightarrow x_{\alpha}$ that is for a given $0<\varepsilon<1$ there is a positive number $N$ such that $\widetilde{M}\left(\left(a_{n}, \beta_{n}\right), x_{a}\right)>(1-\varepsilon)$ for all $n \geq N$. Take $\mathrm{a}_{\beta}=\mathrm{a}_{\mathrm{N}}$, so $\widetilde{\mathrm{M}}\left(\mathrm{a}_{\beta}, \mathrm{x}_{\kappa \varepsilon}\right)>(1-\varepsilon)$. Conversely to prove $\tilde{\mathrm{C}}$ is fuzzy dense in $\tilde{\mathrm{A}}$ we have to show that for each $\mathrm{x}_{\alpha \in} \in \tilde{\mathrm{A}}$ then there is $\mathrm{a}_{\mathrm{k}} \in \widetilde{\mathrm{C}}$ such that $\widetilde{\mathrm{M}}\left(\left(\mathrm{a}_{\mathrm{k}}, \beta_{\mathrm{k}}\right), \mathrm{x}_{\mathrm{c}}\right)>\left(1-\frac{1}{\mathrm{k}}\right)$. Now take $0<\varepsilon<1$ such

that $\frac{1}{\mathrm{k}}<\varepsilon$ for each $\mathrm{k} \geq \mathrm{N}$ for some positive number $\mathrm{N}$. Hence we have a 
sequence $\left(\left(\mathrm{a}_{\mathrm{k}}, \beta_{\mathrm{k}}\right)\right) \in \widetilde{\mathrm{C}}$ such that $\widetilde{\mathrm{M}}\left(\left(\mathrm{a}_{\mathrm{k}}, \beta_{\mathrm{k}}\right), \mathrm{x}_{\alpha}\right)>\left(1-\frac{1}{\mathrm{k}}\right)>(1-\varepsilon)$ for all $\mathrm{k} \geq \mathrm{N}$ that is $\left(\mathrm{a}_{\mathrm{k}}, \beta_{\mathrm{k}}\right) \rightarrow \mathrm{x}_{\alpha \varepsilon}$ so $\mathrm{x}_{\alpha} \in \overline{\mathrm{C}}$

\section{Definition 2.13:}

A sequence $\left\{\left(\mathrm{x}_{\mathrm{n}}, \alpha_{\mathrm{n}}\right)\right\}$ of fuzzy points in a fuzzy distance space $(\tilde{\mathrm{A}}, \widetilde{\mathrm{M}}, *)$ is said to be fuzzy Cauchy if for each $0<\varepsilon<1$ there is a positive number $\mathrm{N}$ such that $\widetilde{\mathrm{M}}\left(\left(\mathrm{x}_{\mathrm{n}}, \alpha_{\mathrm{n}}\right),\left(\mathrm{x}_{\mathrm{m}}, \alpha_{\mathrm{m}}\right)\right)>(1-\varepsilon)$ for all $\mathrm{n}, \mathrm{m} \geq \mathrm{N}$.

\section{Theorem 2.14:}

In a fuzzy distance space every fuzzy convergent sequence of fuzzy points is fuzzy Cauchy.

\section{Proof:}

Let $\left\{\left(\mathrm{x}_{\mathrm{n}}, \alpha_{\mathrm{n}}\right)\right\}$ be a sequence of fuzzy points in a fuzzy distance space $(\tilde{\mathrm{A}}, \widetilde{\mathrm{M}}, *)$ that is fuzzy converges to $\mathrm{x}_{\alpha \varepsilon} \in \tilde{\mathrm{A}}$, then for given $0<\varepsilon<1$ there is a positive number $\mathrm{N}$ such that $\widetilde{\mathrm{M}}\left(\left(\mathrm{x}_{\mathrm{n}}, \alpha_{\mathrm{n}}\right), \mathrm{x}_{\mathrm{c}}\right)>(1-\varepsilon)$. Now by Remark 1.13, there is $(1-r) \in$ $(0,1)$ such that $(1-\varepsilon) *(1-\varepsilon)>(1-r)$. Now for each $m, n \geq N$, we obtain $\widetilde{\mathrm{M}}\left(\left(\mathrm{x}_{\mathrm{m}}, \alpha_{\mathrm{m}}\right),\left(\mathrm{x}_{\mathrm{n}}, \alpha_{\mathrm{n}}\right)\right) \geq \widetilde{\mathrm{M}}\left(\left(\mathrm{x}_{\mathrm{m}}, \alpha_{\mathrm{m}}\right), \mathrm{x}_{\alpha \varepsilon}\right)$ $* \widetilde{\mathrm{M}}\left(\mathrm{x}_{\alpha},\left(\mathrm{x}_{\mathrm{n}}, \alpha_{\mathrm{n}}\right)\right) \geq(1-\varepsilon) *(1-\varepsilon)>(1-\mathrm{r})$. Hence $\left\{\left(\mathrm{x}_{n}, \alpha_{\mathrm{n}}\right)\right\}$ is a fuzzy Cauchy

\section{Proposition 2.15:}

Let $(X, d)$ be a metric space and let $\widetilde{\mathrm{M}}_{d}\left(\mathrm{x}_{\alpha,}, \mathrm{y}_{\beta}\right)=\frac{t}{t+d\left(\mathrm{x}_{\alpha, y_{\beta}}\right)}$ where $t=\min \{\alpha, \beta\}$.

Then $\left\{\left(\mathrm{x}_{n}, \alpha_{n}\right)\right\}$ is a Cauchy sequence in $(\widetilde{\mathrm{A}}, \mathrm{d})$ if and only if $\left\{\left(\mathrm{x}_{\mathrm{n}}, \alpha_{\mathrm{n}}\right)\right\}$ is a fuzzy Cauchy sequence in $(\tilde{\mathrm{A}}$, $\left.\widetilde{\mathrm{M}}_{\mathrm{d}}, *\right)$.

\section{Proof:}

Suppose that $\left\{\left(\mathrm{x}_{\mathrm{n}}, \alpha_{\mathrm{n}}\right)\right\}$ is a Cauchy sequence in $(\tilde{\mathrm{A}}, \mathrm{d})$, then there is a positive number $\mathrm{N}$ such that $\mathrm{d}\left(\left(\mathrm{x}_{\mathrm{m}}, \alpha_{\mathrm{m}}\right)\right.$, $\left.\left(\mathrm{x}_{\mathrm{n}}, \alpha_{\mathrm{n}}\right)\right)<\varepsilon$ for given $\varepsilon$ and for all $\mathrm{m}, \mathrm{n} \geq \mathrm{N}$.

Now $\mathrm{t}+\mathrm{d}\left(\left(\mathrm{x}_{\mathrm{m}}, \alpha_{\mathrm{m}}\right),\left(\mathrm{x}_{\mathrm{n}}, \alpha_{\mathrm{n}}\right)\right)<\mathrm{t}+\varepsilon$, implies $\frac{\mathrm{t}}{\mathrm{t}+\mathrm{d}\left(\left(\mathrm{x}_{\mathrm{m},}, a_{\mathrm{m}}\right),\left(\mathrm{x}_{\mathrm{n},}, \mathrm{c}_{\mathrm{n}}\right)\right)}>\frac{\mathrm{t}}{\mathrm{t}+\mathrm{\varepsilon}}$ Put $\frac{\mathrm{t}}{\mathrm{t}+\varepsilon}=(1-\mathrm{r})$ for some $0<\mathrm{r}<1$. It follows that $\widetilde{\mathrm{M}}_{\mathrm{d}}\left(\left(\mathrm{x}_{\mathrm{m}}, \alpha_{\mathrm{m}}\right),\left(\mathrm{x}_{\mathrm{n}}, \alpha_{\mathrm{n}}\right)\right)>(1-\mathrm{r})$ for all $\mathrm{m}, \mathrm{n} \geq \mathrm{N}$. Hence $\left\{\left(\mathrm{x}_{\mathrm{n}}, \alpha_{\mathrm{n}}\right)\right\}$ is a fuzzy Cauchy sequence in $(\tilde{\mathrm{A}}$, $\left.\widetilde{\mathrm{M}}_{\mathrm{d}}, *\right)$.

Conversely, assume that $\left\{\left(\mathrm{x}_{\mathrm{n}}, \alpha_{\mathrm{n}}\right)\right\}$ is a fuzzy Cauchy sequence in $\left(\tilde{\mathrm{A}}, \widetilde{\mathrm{M}}_{\mathrm{d}}, *\right)$ then given $0<\varepsilon<1$, there is a positive number $\mathrm{N}$ such that $\widetilde{\mathrm{M}}_{\mathrm{d}}\left(\left(\mathrm{x}_{\mathrm{m}}, \alpha_{\mathrm{m}}\right),\left(\mathrm{x}_{\mathrm{n}}, \alpha_{\mathrm{n}}\right)\right)>(1-\varepsilon)$. Put $(1-\varepsilon)=\mathrm{r}$ then $\frac{\mathrm{t}}{\mathrm{t}+\mathrm{d}\left(\left(\mathrm{x}_{\mathrm{m},}, \alpha_{\mathrm{m}}\right),\left(\mathrm{x}_{\mathrm{n}}, \alpha_{\mathrm{n}}\right)\right)}>\mathrm{r}$ for all $\mathrm{n}, \mathrm{m} \geq \mathrm{N}$.

This implies $\mathrm{t}+\mathrm{d}\left(\left(\mathrm{x}_{\mathrm{m},}, \alpha_{\mathrm{m}}\right),\left(\mathrm{x}_{\mathrm{n}}, \alpha_{\mathrm{n}}\right)\right)<\frac{\mathrm{t}}{\mathrm{r}}$, it follows that $\mathrm{d}\left(\left(\mathrm{x}_{\mathrm{m}}, \alpha_{\mathrm{m}}\right),\left(\mathrm{x}_{\mathrm{n}}, \alpha_{\mathrm{n}}\right)\right)<\left(\frac{\mathrm{t}}{\mathrm{r}}-\mathrm{t}\right)$ for all $\mathrm{n}, \mathrm{m} \geq \mathrm{N}$, put $\frac{\mathrm{t}}{\mathrm{r}}-$ $\mathrm{t}=\mathrm{k}$. Then $\mathrm{d}\left(\left(\mathrm{x}_{\mathrm{m}}, \alpha_{\mathrm{m}}\right),\left(\mathrm{x}_{\mathrm{n}}, \alpha_{\mathrm{n}}\right)\right)<\mathrm{k}$ for all $\mathrm{n}, \mathrm{m} \geq \mathrm{N}$. Hence $\left\{\left(\mathrm{x}_{\mathrm{m}}, \alpha_{\mathrm{m}}\right)\right\}$ is Cauchy sequence in $(\tilde{\mathrm{A}}, \mathrm{d})$

\section{Definition 2.16:}

Let $\left\{\left(x_{n}, \alpha_{n}\right)\right\}$ be a given sequence of fuzzy points in a fuzzy distance space $(\tilde{A}, \widetilde{M}, *)$ and let $\left(n_{k}\right)$ be a sequence of positive integer such that $n_{1}<n_{2}<n_{3}<\ldots$ Then the sequence $\left\{\left(x_{n_{k}}, \alpha_{n_{k}}\right)\right\}$ is called a subsequence of $\left\{\left(x_{n}, \alpha_{n}\right)\right\}$. If $\left(x_{n_{k}}, \alpha_{n_{k}}\right)$ fuzzy converges, its limit is called a sub sequential limit of $\left\{\left(x_{n}, \alpha_{n}\right)\right\}$.lt is clear that a sequence $\left\{\left(x_{n}, \alpha_{n}\right)\right\}$ in $\tilde{A}$ fuzzy converges to $\mathrm{x}_{\alpha}$ if and only if every subsequence of it fuzzy converges to $\mathrm{x}_{\alpha}$.

\section{Proposition 2.17:}

If a fuzzy Cauchy sequence of fuzzy points in a fuzzy distance space $(\tilde{A}, \widetilde{\mathrm{M}}, *)$ contains a fuzzy convergent subsequence, then the sequence fuzzy converges to the same fuzzy limit as thesubsequence. 


\section{Proof:}

Let $\left\{\left(\mathrm{x}_{\mathrm{n}}, \alpha_{\mathrm{n}}\right)\right\}$ be a fuzzy Cauchy sequence in $(\tilde{\mathrm{A}}, \widetilde{\mathrm{M}}, *)$. Then for a given $0<\varepsilon<1$, there exists an integer $\mathrm{N}$ such that $\widetilde{\mathrm{M}}\left(\left(\mathrm{x}_{\mathrm{m}}, \alpha_{\mathrm{m}}\right),\left(\mathrm{x}_{\mathrm{n}}, \alpha_{\mathrm{n}}\right)\right)>(1-\mathrm{\varepsilon})$ whenever $\mathrm{m}, \mathrm{n} \geq \mathrm{N}$. Denote by $\left\{\left(\mathrm{x}_{\mathrm{n}_{\mathrm{k}}}, \alpha_{\mathrm{n}_{\mathrm{k}}}\right)\right\}$ a fuzzy convergent subsequence of $\left\{\left(\mathrm{x}_{\mathrm{n}}, \alpha_{\mathrm{n}}\right)\right\}$ and its limit by $\mathrm{x}_{\alpha \cdot}$. It follows that $\widetilde{\mathrm{M}}\left(\left(\mathrm{x}_{\mathrm{n}_{\mathrm{m}}}, \alpha_{\mathrm{n}_{\mathrm{m}}}\right),\left(\mathrm{x}_{\mathrm{n}}, \alpha_{\mathrm{n}}\right)\right)>(1-\varepsilon)$ whenever $\mathrm{m}, \mathrm{n} \geq \mathrm{N}$. Since $\left(\mathrm{n}_{\mathrm{k}}\right)$ is strictly increasing sequence of positive integer.

Now $\widetilde{\mathrm{M}}\left(\mathrm{x}_{\alpha},\left(\mathrm{x}_{\mathrm{n}}, \alpha_{\mathrm{n}}\right)\right) \geq \widetilde{\mathrm{M}}\left(\mathrm{x}_{\alpha},\left(\mathrm{x}_{\mathrm{n}_{\mathrm{m}}}, \alpha_{\mathrm{n}_{m}}\right)\right) * \widetilde{\mathrm{M}}\left(\left(\mathrm{x}_{\mathrm{n}_{\mathrm{m}}}, \alpha_{\mathrm{n}_{m}}\right),\left(\mathrm{x}_{\mathrm{n}}, \alpha_{\mathrm{n}}\right)\right)>\widetilde{\mathrm{M}}\left(\mathrm{x}_{\alpha},\left(\mathrm{x}_{\mathrm{n}_{\mathrm{m}}}, \alpha_{\mathrm{n}_{m}}\right)\right) *(1-\varepsilon)$ Letting $\mathrm{m} \rightarrow \infty$, we have $\widetilde{\mathrm{M}}\left(\mathrm{x}_{\alpha},\left(\mathrm{x}_{\mathrm{n}}, \alpha_{\mathrm{n}}\right)\right) \geq 1 *(1-\varepsilon)=(1-\varepsilon)$ So, the sequence $\left\{\left(\mathrm{x}_{\mathrm{n}}, \alpha_{\mathrm{n}}\right)\right\}$ fuzzy converges to $\mathrm{x}_{\alpha}$

\section{Definition 2.18:}

Let $(\tilde{A}, \widetilde{M}, *)$ be a fuzzy distance space. A fuzzy subset $\widetilde{\mathrm{C}}$ of $\tilde{\mathrm{A}}$ is said to be fuzzy bounded if there exists $0<\mathrm{r}<1$ such that, $\widetilde{\mathrm{M}}\left(\mathrm{x}_{\alpha}, \mathrm{y}_{\beta}\right)>(1-\mathrm{r})$, for all $\mathrm{x}_{\alpha}, \mathrm{y}_{\beta} \in \tilde{\mathrm{C}}$.

\section{Proposition 2.19:}

Let $(X, d)$ be a metric space and let $\widetilde{\mathrm{M}}_{d}\left(\mathrm{x}_{\alpha}, \mathrm{Y}_{\beta}\right)=\frac{t}{t+d\left(\mathrm{x}_{\alpha} y_{\beta}\right)}$ where $t=\alpha \wedge \beta$ then a fuzzy subset $\tilde{\mathrm{C}}$ of $\tilde{\mathrm{A}}$ is fuzzy bounded if and only if it is bounded.

\section{Proof:}

Assume that $\widetilde{\mathrm{C}}$ is fuzzy bounded then there is $0<r<1$ such that $\widetilde{\mathrm{M}}_{\mathrm{d}}\left(\mathrm{x}_{\alpha}, \mathrm{y}_{\beta}\right)>(1-r)$ for all $\mathrm{x}_{\alpha}, \mathrm{y}_{\beta} \in \widetilde{\mathrm{C}}$. Now put $(1-r)=\varepsilon$ Then $\widetilde{\mathrm{M}}_{\mathrm{d}}\left(\mathrm{x}_{\omega}, \mathrm{y}_{\beta}\right)=\frac{\mathrm{t}}{\mathrm{t}+\mathrm{d}\left(\mathrm{x}_{\alpha}, y_{\beta}\right)}>\varepsilon$. Implies $\mathrm{t}+\mathrm{d}\left(\mathrm{x}_{\alpha}, \mathrm{y}_{\beta}\right)<\frac{\mathrm{t}}{\varepsilon}$, it follows that $\mathrm{d}\left(\mathrm{x}_{\alpha}, \mathrm{y}_{\beta}\right)<\frac{\mathrm{t}}{\mathrm{s}}-\mathrm{t}$, put $\frac{\mathrm{t}}{\mathrm{s}}-\mathrm{t}=\mathrm{k}$. Therefore $\mathrm{d}\left(\mathrm{x}_{\alpha}, \mathrm{y}_{\beta}\right)<\mathrm{k}$ for all $\mathrm{x}_{\alpha}, \mathrm{y}_{\beta} \in \tilde{\mathrm{C}}$. Hence $\tilde{\mathrm{C}}$ is bounded.

Conversely, suppose that $\tilde{\mathrm{C}}$ is bounded then there is $\mathrm{k}$ such that $\mathrm{d}\left(\mathrm{x}_{\alpha}, \mathrm{y}_{\beta}\right)<\mathrm{k}$ for all $\mathrm{x}_{\alpha}, \mathrm{y}_{\beta} \in \widetilde{\mathrm{C}}$. Implies $\mathrm{t}+\mathrm{d}\left(\mathrm{x}_{\alpha}, \mathrm{y}_{\beta}\right)<$ $\mathrm{t}+\mathrm{k}$, implies $\frac{\mathrm{t}}{\mathrm{t}+\mathrm{d}\left(\mathrm{x}_{\alpha} y_{\beta}\right)}>\frac{\mathrm{t}}{\mathrm{t}+\mathrm{k}}$. Let $0<\varepsilon<1$ with $\frac{\mathrm{t}}{\mathrm{t}+\mathrm{k}}=(1-\varepsilon)$. Therefore $\left.\widetilde{\mathrm{M}}_{\mathrm{d}}\left(\mathrm{x}_{\alpha}, \mathrm{y}_{\beta}\right)\right)>(1-\varepsilon)$ for all $\mathrm{x}_{\alpha}, \mathrm{y}_{\beta} \in \tilde{\mathrm{C}}$ Hence $\widetilde{C}$ is fuzzy bounded.

\section{Lemma 2.20:}

A fuzzy convergent sequence of fuzzy points in a fuzzy distance space $(\tilde{A}, \widetilde{M}, *)$ is fuzzy bounded and its fuzzy limit is unique.

\section{Proof:}

Suppose that $\left\{\left(\mathrm{x}_{\mathrm{n}}, \alpha_{\mathrm{n}}\right)\right\}$ fuzzy converges to $\mathrm{x}_{\alpha \varepsilon}$ then given $0<\varepsilon<1$ we can find a positive number $\mathrm{N}$ such that $\widetilde{\mathrm{M}}$ $\left(\left(\mathrm{x}_{\mathrm{n}}, \alpha_{\mathrm{n}}\right), \mathrm{x}_{\alpha}\right)>(1-\varepsilon)$ for all $\mathrm{n} \geq \mathrm{N}_{-}$Let $\left.\left.\left.\mathrm{t}=\min \left\{\widetilde{\mathrm{M}}_{(}\left(\mathrm{x}_{1}, \alpha_{1}\right), \mathrm{x}_{\alpha \varepsilon}\right), \widetilde{\mathrm{M}}\left(\mathrm{x}_{2}, \alpha_{2}\right), \mathrm{x}_{\alpha \varepsilon}\right), \ldots, \widetilde{\mathrm{M}}_{(}\left(\mathrm{x}_{\mathrm{N}}, \alpha_{\mathrm{N}}\right), \mathrm{x}_{\alpha}\right)\right\}$. Then by Remark 1.13 there is_ $0<r<1$ such that $t *(1-\varepsilon)>(1-r)$. Now for all $n \geq N \widetilde{M}\left(\left(x_{n}, \alpha_{n}\right), x_{\alpha}\right) \geq \widetilde{M}\left(\left(x_{n}, \alpha_{n}\right)\right.$, $\left(\mathrm{x}_{\mathrm{N}}, \alpha_{\mathrm{N}}\right) * \widetilde{\mathrm{M}}\left(\left(\mathrm{x}_{\mathrm{N}}, \alpha_{\mathrm{N}}\right), \mathrm{x}_{\alpha}\right) \geq \mathrm{t} *(1-\varepsilon)>(1-\mathrm{r})$.

Hence $\left\{\left(\mathrm{x}_{\mathrm{n}}, \alpha_{\mathrm{n}}\right)\right\}$ is fuzzy bounded.

Assume that $\quad\left(x_{n}, \alpha_{n}\right) \rightarrow x_{\alpha}$ and $\left(x_{n}, \alpha_{n}\right) \rightarrow y_{\beta}$. So $\lim _{n \rightarrow \infty} \widetilde{M}\left(\left(x_{n}, \alpha_{n}\right), x_{\alpha}\right)=1$ and $\lim _{n \rightarrow \infty} M\left(\left(x_{n}, \alpha_{n}\right), y_{\beta}\right)=$ 1. Now $\widetilde{\mathrm{M}}\left(\mathrm{x}_{\alpha}, \mathrm{y}_{\beta}\right) \geq \widetilde{\mathrm{M}}\left(\mathrm{x}_{\alpha},\left(\mathrm{x}_{\mathrm{n}}, \alpha_{\mathrm{n}}\right)\right) * \widetilde{\mathrm{M}}\left(\left(\mathrm{x}_{\mathrm{n}}, \alpha_{\mathrm{n}}\right), \mathrm{y}_{\beta}\right)$ Taking limit to both sides, as $\mathrm{n}$ tends to $\infty$, we obtain $\widetilde{\mathrm{M}}\left(\mathrm{x}_{\alpha}, \mathrm{y}_{\beta}\right) \geq 1 * 1=1$. So $\widetilde{\mathrm{M}}\left(\mathrm{x}_{\alpha}, \mathrm{y}_{\beta}\right)=1$, hence $\mathrm{x}_{\alpha}=\mathrm{y}_{\beta}$

Definition 2.21: Let $(\tilde{\mathrm{A}}, \widetilde{\mathrm{M}}, *)$ be a fuzzy distance space, then we define a fuzzy closed fuzzy ball with center $\mathrm{x}_{\alpha \kappa} \in \tilde{\mathrm{A}}$ and radius $r, 0<r<1$ by $\widetilde{B}\left[x_{\alpha c}, r\right]=\left\{y_{\beta} \in X: \widetilde{M}\left(x_{\alpha^{u}}, y_{\beta}\right) \geq(1-r)\right\}$. 


\section{Lemma 2.22:}

Every fuzzy closed fuzzy ball in a fuzzy distance space $(\tilde{A}, \widetilde{M}, *)$ is fuzzy closed fuzzy set.

\section{Proof:}

Let $\mathrm{y}_{\beta} \in \widetilde{\mathrm{B}}\left[\mathrm{x}_{\alpha}, \mathrm{r}\right]$ then by lemma 2.11 there exists a sequence $\left\{\left(\mathrm{y}_{\mathrm{n}}, \beta_{\mathrm{n}}\right)\right\}$ in $\widetilde{\mathrm{B}}\left[\mathrm{x}_{\alpha}, r\right]$ such that $\left(\mathrm{y}_{\mathrm{n}}, \beta_{\mathrm{n}}\right)$ converges to $\mathrm{y}_{\beta}$ ,therefore $\quad \lim _{\mathrm{n} \rightarrow \infty} \widetilde{\mathrm{M}}\left(\left(\mathrm{y}_{\mathrm{n}}, \beta_{\mathrm{n}}\right), \mathrm{y}_{\beta}\right)=1$ Now, $\widetilde{\mathrm{M}}\left(\mathrm{x}_{\alpha}, \mathrm{y}_{\beta}\right) \geq \widetilde{\mathrm{M}}\left(\mathrm{x}_{\alpha},\left(\mathrm{y}_{\mathrm{n}}, \beta_{\mathrm{n}}\right) \quad\right.$ ) * $\widetilde{\mathrm{M}}\left(\left(\mathrm{y}_{\mathrm{n}}, \beta_{\mathrm{n}}\right), \mathrm{y}_{\beta}\right)$ $\geq \lim _{n \rightarrow \infty} \widetilde{\mathrm{M}}\left(\mathrm{x}_{\alpha},\left(\mathrm{y}_{\mathrm{n}}, \beta_{\mathrm{n}}\right)\right) * \lim _{\mathrm{n} \rightarrow \infty} \widetilde{\mathrm{M}}\left(\left(\mathrm{y}_{\mathrm{n}}, \beta_{\mathrm{n}}\right), \mathrm{y}_{\beta}\right)>(1-r) * 1=(1-r)$ Hence $\mathrm{y}_{\beta} \in \widetilde{\mathrm{B}}\left[\mathrm{x}_{\alpha}, r\right]$, therefore $\widetilde{\mathrm{B}}\left[\mathrm{x}_{\alpha}, r\right]$ is a fuzzy closed fuzzy set

\section{Theorem 2.23:}

A fuzzy distance space is a fuzzy topological space.

\section{Proof:}

Let $(\tilde{\mathrm{A}}, \widetilde{\mathrm{M}}, *)$ be a fuzzy distance space . Define $\tau_{\widetilde{\mathrm{M}}}=\left\{\widetilde{\mathrm{C}} \subset \tilde{\mathrm{A}}: \mathrm{x}_{\mathrm{\alpha}} \in \widetilde{\mathrm{C}}\right.$ if and only if there exists $0<\mathrm{r}<1$ such that $\widetilde{\mathrm{B}}\left(\mathrm{x}_{\alpha \varepsilon}\right.$, r) $\subset \widetilde{\mathrm{C}}\}$.We prove now $\tau_{\mathrm{M}}$ is a fuzzy topology on $\tilde{A}$.

(i) Clearly $\phi$ and $\tilde{A}$ belong to $\tau_{\mathrm{M}}$.

(ii) Let $\widetilde{\mathrm{C}}_{1}, \widetilde{\mathrm{C}}_{2}, \ldots ., \widetilde{\mathrm{C}}_{\mathrm{n}} \in \tau_{\mathrm{M}}$ and put $\mathrm{U}=\mathrm{n}_{\mathrm{i}=1}^{\mathrm{n}} \widetilde{\mathrm{C}}_{\mathrm{i}}$. We shall show that $\mathrm{U} \in \tau_{\mathrm{M}}$.

Let $\mathrm{a}_{\alpha \in} \in \mathrm{U}$ then $\mathrm{a}_{\alpha} \in \widetilde{\mathrm{C}}_{\mathrm{i}}$ for each $1 \leq \mathrm{i} \leq \mathrm{n}$. Hence there exists $0 \leq \mathrm{r}_{\mathrm{i}} \leq 1$ such that $\widetilde{\mathrm{B}}\left(\mathrm{a}_{\alpha}, \mathrm{r}_{\mathrm{i}}\right) \subset \widetilde{\mathrm{C}}_{\mathrm{i}}$.

Let $\mathrm{r}=\min \left\{\mathrm{r}_{\mathrm{i}}: 1 \leq \mathrm{i} \leq \mathrm{n}\right\}$ thus $\mathrm{r} \leq \mathrm{r}_{\mathrm{i}}$ for all $1 \leq \mathrm{i} \leq \mathrm{n}$ so $(1-\mathrm{r}) \geq\left(1-\mathrm{r}_{\mathrm{i}}\right)$ for all $1 \leq \mathrm{i} \leq \mathrm{n}$. So $\widetilde{\mathrm{B}}\left(\mathrm{a}_{a}, \mathrm{r}\right) \subseteq \widetilde{\mathrm{C}}_{\mathrm{i}}$ for all $1 \leq \mathrm{i} \leq \mathrm{n}$

Therefore $\widetilde{\mathrm{B}}\left(\mathrm{a}_{\alpha}, \mathrm{r}\right) \subseteq \cap_{\mathrm{i}=1}^{\mathrm{n}} \widetilde{\mathrm{C}}_{\mathrm{i}}=\mathrm{U}$, this shows that $\mathrm{U}_{\in} \tau_{\mathrm{M}}$.

(iii) Let $\left\{\widetilde{\mathrm{C}}_{\mathrm{i}}: \mathrm{i} \in \mathrm{l}\right\} \in \tau_{\mathrm{M}}$ and put $\widetilde{\mathrm{V}}=\mathrm{U}_{\mathrm{i} \in \mathrm{I}} \tilde{\mathrm{C}}_{\mathrm{i}}$. We shall show that $\widetilde{\mathrm{V}} \in \tau_{\mathrm{M}}$.

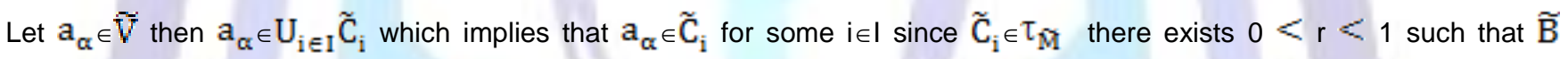
$\left(\mathrm{a}_{\alpha}, \mathrm{r}\right) \subset \widetilde{\mathrm{C}}_{\mathrm{i}}$

Hence $\widetilde{\mathrm{B}}\left(\mathrm{a}_{a}, r\right) \subset \widetilde{\mathrm{C}}_{\mathrm{i}} \subseteq \mathrm{U}_{\mathrm{i} \in \mathrm{I}} \widetilde{\mathrm{C}}_{\mathrm{i}}=\widetilde{\mathrm{V}}$, this shows that $\widetilde{\mathrm{V}} \in \tau_{\mathbb{\mathrm { M }}}$.

Hence $\left(\tilde{\mathrm{A}}, \tau_{\mathrm{M}}\right)$ is a fuzzy topological space. $\tau_{\mathbb{\mathrm { M }}}$ is called the fuzzy topology induced by $\widetilde{\mathrm{M}}$

\section{Proposition 2.24:}

Let $(X, d)$ be an ordinary metric space. Then $(\tilde{A}, d)$ is a metric space and let $\widetilde{M}_{d}\left(x_{\alpha}, y_{\beta}\right)=\frac{t}{t+d\left(x_{\alpha}, y_{\beta}\right)}$

be the fuzzy distance space induced by $d$. Then the topology $\tau_{d}$ induced by $d$ and the fuzzy topology $\tau_{\mathbb{M}_{d}}$ induced by $\widetilde{\mathrm{M}}_{d}$ are the same. That is $\tau_{\mathrm{d}}=\tau_{\mathrm{N}_{\mathrm{d}}}$.

\section{Proof:}

Suppose that $\widetilde{\mathrm{C}} \in \tau_{\mathrm{d}}$ then there exists $0<\varepsilon<1$ such that $\widetilde{\mathrm{B}_{\varepsilon}}\left(\mathrm{x}_{\mathrm{c}}\right) \subseteq \widetilde{\mathrm{C}}$ for every $\mathrm{x}_{\alpha} \in \widetilde{\mathrm{C}}$, we obtain $\widetilde{\mathrm{M}}_{\mathrm{d}}\left(\mathrm{x}_{\alpha^{\prime}}, \mathrm{y}_{\beta}\right)=\frac{\mathrm{t}}{\mathrm{t}+\mathrm{d}\left(\mathrm{x}_{\alpha}, y_{\beta}\right)}>\frac{\mathrm{t}}{\mathrm{t}+\varepsilon}$ where $\mathrm{t}=\alpha \wedge \beta$. Let $1-\mathrm{r}=\frac{\mathrm{t}}{\mathrm{t}+\varepsilon}$, then $\widetilde{\mathrm{M}}_{\mathrm{d}}\left(\mathrm{x}_{\alpha^{\prime}}, \mathrm{y}_{\beta}\right)>(1-r)$. It follows that $\widetilde{\mathrm{B}}\left(\mathrm{x}_{\alpha}, r\right) \subseteq \widetilde{\mathrm{C}}$. Hence $\widetilde{\mathrm{C}}_{\in} \tau_{\mathrm{M}_{\mathrm{d}}}$. This shows that $\tau_{\mathrm{d} \subseteq} \tau_{\mathrm{M}_{\mathrm{d}}}$.

Conversely, suppose that $\widetilde{\mathrm{C}} \in \tau_{\mathbb{N}_{d}}$ then there exists $0<r<1$ such that $\widetilde{\mathrm{B}}\left(\mathrm{x}_{\alpha}, \mathrm{r}\right) \subseteq \widetilde{\mathrm{C}}$ for every $\mathrm{x}_{\alpha \varepsilon} \in \widetilde{\mathrm{C}}$. 
Now $\widetilde{M}_{d}\left(x_{\alpha}, y_{\beta}\right)=\frac{t}{t+d\left(x_{\alpha} y_{\beta}\right)}>(1-r)$ which implies that $t>t(1-r)+(1-r) d\left(x_{\alpha}, y_{\beta}\right)$. Then $d\left(x_{\alpha}, y_{\beta}\right)<\frac{r}{1-r}$ Let $\quad \boldsymbol{\varepsilon}=\frac{\mathrm{r}}{(1-\mathrm{r})}$ then $\mathrm{d}\left(\mathrm{x}_{\alpha^{p}}, \mathrm{y}_{\beta}\right)<\varepsilon$ and therefore $\widetilde{\mathrm{B}}_{\varepsilon}\left(\mathrm{x}_{\alpha}\right) \subseteq \widetilde{\mathrm{C}}$.

Hence $\tilde{\mathrm{C}} \in \tau_{\mathrm{d}}$. This implies that $\tau_{\mathrm{M}_{\mathrm{d}}} \subseteq \tau_{\mathrm{d}}$, therefore $\tau_{\mathrm{d}}=\tau_{\mathrm{M}_{\mathrm{d}}}$

\section{Proposition 2.25:}

Let $(X, d)$ be an ordinary metric space. Then $(\widetilde{\mathbb{A}}, \mathrm{d})$ is a metric space and $\widetilde{\mathrm{R}}_{\mathrm{d}}=\left\{\left(\mathrm{x}_{\mathrm{n}}, \alpha_{\mathrm{n}}\right)\right\}$ and $\left\{\left(\hat{\mathbb{x}}_{\mathrm{n}},{ }^{\prime} \alpha_{\mathrm{n}}\right)\right\}$ fuzzy Cauchy sequences in $\left.(\mathrm{X}, \mathrm{d}),\left(\mathrm{x}_{\mathrm{n}}, \alpha_{\mathrm{n}}\right) \sim\left(\hat{\mathrm{x}}_{\mathrm{n}}^{\prime},{ }^{\prime} \alpha_{\mathrm{n}}\right) \Leftrightarrow \lim _{\mathrm{n} \rightarrow \infty} \mathrm{d}\left(\left(\mathrm{x}_{\mathrm{n}}, \alpha_{\mathrm{n}}\right),\left(\hat{\mathrm{x}}_{\mathrm{n}},{ }^{\prime} \alpha_{\mathrm{n}}\right)\right)=0\right\}$

$. \widetilde{\mathrm{R}}_{\mathbb{\mathrm { A }}_{\mathrm{d}}}=\left\{\left(\mathrm{x}_{\mathrm{n}}, \alpha_{\mathrm{n}}\right)\right\}$ and $\left\{\left(\mathbb{\mathrm { x }}_{\mathrm{n}},{ }^{\prime} \alpha_{\mathrm{n}}\right)\right\}$ fuzzy Cauchy sequences in $\left(\mathrm{X}, \widetilde{\mathrm{M}}_{\mathrm{d}}, *\right)$ such that $\left(\mathrm{x}_{\mathrm{n}}, \alpha_{\mathrm{n}}\right) \sim\left(\mathbb{\mathrm { x }}_{\mathrm{n}},{ }^{\prime} \alpha_{\mathrm{n}}\right)$ $\left.\Leftrightarrow \lim _{\mathrm{n} \rightarrow \infty} \widetilde{\mathrm{M}}_{\mathrm{d}}\left(\left(\mathrm{x}_{\mathrm{n}}, \alpha_{\mathrm{n}}\right),\left(\tilde{\mathrm{x}}_{\mathrm{n}},{ }^{\prime} \alpha_{\mathrm{n}}\right)\right)=1\right\}$. Then $\widetilde{\mathrm{R}}_{\mathrm{d}}=\widetilde{\mathrm{R}}_{\mathrm{M}_{\mathrm{d}}}$.

\section{Proof:}

The prove follows from the fact $\lim _{\mathrm{n} \rightarrow \infty} \mathrm{d}\left(\left(\mathrm{x}_{\mathrm{n}}, \alpha_{\mathrm{n}}\right),\left(\hat{\mathrm{x}}_{\mathrm{n}},{ }^{o} \alpha_{\mathrm{n}}\right)\right)=0 \Leftrightarrow \lim _{\mathrm{n} \rightarrow \infty} \widetilde{\mathrm{M}}_{\mathrm{d}}\left(\left(\mathrm{x}_{\mathrm{n}}, \alpha_{\mathrm{n}}\right),\left(\hat{\mathrm{x}}_{\mathrm{n}},{ }^{n} \alpha_{\mathrm{n}}\right)\right)=$

\section{Theorem 2.26:}

Every fuzzy distance space on a fuzzy set is a fuzzy Hausdorff space.

\section{Proof:}

Let $(\tilde{A}, \widetilde{M}, *)$ be a fuzzy distance space and let $x_{\alpha}, y_{\beta} \in \tilde{A}$ with $x_{\alpha} \neq y_{\beta}$ Let $\widetilde{M}\left(x_{\alpha}, y_{\beta}\right)=r \quad$ for some $0<r$ $<1$. Then for each $t, r<t<1$, we can find $r_{1}$ such that $r_{1} * r_{1} \geq t$ by Remark 1.13. Now consider the two fuzzy open fuzzy balls $\widetilde{\mathrm{B}}\left(\mathrm{x}_{\kappa}, 1-\mathrm{r}_{1}\right)$ and

$\widetilde{\mathrm{B}}\left(\mathrm{y}_{\beta}, 1-\mathrm{r}_{1}\right)$.Then $\widetilde{\mathrm{B}}\left(\mathrm{x}_{\alpha}, 1-\mathrm{r}_{1}\right) \cap \widetilde{\mathrm{B}}\left(\mathrm{y}_{\beta}, 1-\mathrm{r}_{1}\right)=\emptyset$ Since if there exists $\mathrm{z}_{\sigma} \in \widetilde{\mathrm{B}}\left(\mathrm{x}_{\alpha}, 1-\mathrm{r}_{1}\right) \cap \widetilde{\mathrm{B}}\left(\mathrm{y}_{\beta}, 1-\mathrm{r}_{1}\right)$.

Then $r=\widetilde{\mathrm{M}}\left(\mathrm{x}_{\alpha}, \mathrm{y}_{\beta}\right) \geq \widetilde{\mathrm{M}}\left(\mathrm{x}_{\alpha}, \mathrm{z}_{\sigma}\right) * \widetilde{\mathrm{M}}\left(\mathrm{z}_{\sigma}, \mathrm{y}_{\beta}\right) \geq \mathrm{r}_{1} * \mathrm{r}_{1} \geq \mathrm{t}>\mathrm{r}$, which is a contradiction, therefore

$(\tilde{A}, \widetilde{M}, *)$ is a fuzzy Hausdorff space

\section{REFERENCES}

[1] Company, C.F. Romaguera, S. and Tirado, P, "The Bicompletion of Fuzzy Quasi metric spaces" , Fuzzy Sets and Systems , Vol.166 (2011) 56-64.

[2] Dersanambika, K.S. and Aswathy, M.R., "Fixed point theorem in fuzzy metric spaces" , Int.J. contemp Math. Sciences , Vol.6,No.22(2011)1079-1089.

[3] Dubbois, D. and Prade, H." Fuzzy sets and systems, Theory and Application " , Academic Press , Inc. (1980).

[4] Hassan, A.G.," Pseudo- Complete fuzzy locally convex algebras " Ph.D. thesis , AL-Mustansirya University , Baghdad 2002.

[5] George, A. and Veeramani, P."On some results in fuzzy metric spaces", Fuzzy Sets and Systems, Vol.64(1994) 395399 .

[6] Gregori, V. and Romaguera, S. , "Some properties of fuzzy metric spaces ", Fuzzy Sets and Systems , Vol.115(2000)485-489.

[7] Gregori , V., Romaguera , S. and sapena ,A. ,"Uniform continuity in fuzzy metric spaces" ,Rend. Ist. Mat. Univ. trieste suppl.2,Vol.32(2001) 81-88.

[8] Gregori, V. and Sapena, A."On fixed point theorems in fuzzy metric spsces", Fuzzy Sets and Systems , Vol.125(2002) 245-253 .

[9] Gregori, V., Romaguera, S. and Sapena,A.,"A characterization of bicomppletable fuzzy quasi metric spaces " , Fuzzy Sets and Systems, Vol. 152(2005) 395-402. 
[10] Gregori,V., Minana, J. and Morillas, S. , "Some question in fuzzy metric spaces " , Fuzzy Sets and Systems Vol.204(2012)71-85 .

[11] Kider, J., "Fuzzy Locally convex *-Algebras" Ph.D. thesis, University of Technology, 2004.

[12]Klir, G. and Folger, T., "fuzzy sets Uncertainty and information ", prentice, Hall International , Inc (1988) .

[13] Kider, J. ,"Compact standard fuzzy metric space", International J. of Math. Archive , Vol .5 No.7 (2014) $129-136$.

[14] Kramosil, D. and Michalek, J., "Fuzzy metric and statistical metric spaces, Kybernetika, Vol.11 (1975) $326-334$.

[15] Lopez, R. and Romaguera, S. "The Hausdorff fuzzy metric on compact sets ",Fuzzy Sets and System , Vol.147(2004) 273-283 .

[16]Mihet, D. "A Banach Contraction theorem in fuzzy metric spaces ", Fuzzy Sets and System Vol.144(2004) 431-439 .

[17] Pao-Ming, P. and Ying-Ming, L, "Fuzzy Topology II, products and Quotient spaces " , J. Math Anal. Appl.Vol.77(1980) 20-37.

[18] Rudin , W., "Functional Analysis"., Tata Mcgraw - Hill publishing company Itd , 1974 .

[19] Wong, C."Fuzzy points and local properties of fuzzy Topology , J. Math .Anal . Appl., Vol.46(1974) 316-328 .

[20] Zadeh, L."Fuzzy sets "Information and control, Vol.8(1965) 338-353. 